\title{
Advances in gas turbine blade cooling technology
}

\author{
R. S. Amano \\ University of Wisconsin-Milwaukee, USA
}

\begin{abstract}
This article presents a background of the gas turbine blade cooling technologies along with numerical methodologies and physical models that are most commonly used in the computations of blade flows in gas turbine blades. In addition some advancement in the cooling technologies is also discussed.

Keywords: high temperature flow field, gas turbine, advanced cooling technology.
\end{abstract}

\section{Introduction}

To increase the efficiency and the power of modern power plant gas turbines, designers are continually trying to raise the maximum turbine inlet temperature. Over the last decade the temperature has risen from $1500 \mathrm{~K}$ to $1750 \mathrm{~K}$ in some high-performance units. With this increase of the temperature only about $25 \%$ can be attributed to improved alloys. New materials, such as ceramics, could help increase this maximum temperature even more in the future. However, most of the recent improvements in inlet temperature come from better cooling of the blades and a greater understanding of the heat transfer and the threedimensional temperature distribution in the turbine passage. Higher gas temperature generally causes increased blade temperature and greater temperature gradients, both of which can have a detrimental effect on service life. As of today improvements in computational techniques in turbomachines have been attempted by industrial researchers because the numerical approaches are quite advantageous in comparison with experimentation, due to its ease of modeling, relatively complicated geometry and an unsteady flow nature. In fact, modern turbomachinery operates under extremely complex three-dimensional flow conditions, and further improvement in performance requires detailed 
knowledge of the flow structure. Particularly, the need to estimate operating conditions, secondary flows, and turbulence and heat transfer rates demands that viscous models be examined. Near the hub and tip of a turbine stator/rotor passage, the flow is affected by the interaction between the stream-wise boundary layer and the side-wall boundary layer. Although this region is thin, its effect on the overall aerodynamic performance cannot be neglected. To design a high performance turbine, an engineer has to understand the detailed threedimensional flow field near the hub and tip. The flow near the midspan of a turbine stator passage is, principally, driven by the inviscid process. However, some important characteristics and flow parameters are strongly influenced by the turbulence transport near the solid walls and the wake region behind the airfoils. Under certain operating conditions, the boundary layer development on the blade surface is much enhanced due to the existence of adverse pressure gradients, which have considerable effect on the following rotor stage. For the reasons mentioned above, a technique for performing three-dimensional computations becomes more and more necessary. In the past decade computational fluid dynamics (CFD) has undergone significant evolution in turbomachinery research. Coincident with the substantial enhancements in computer technologies, three-dimensional computations became more and more useful.

This paper summarizes the most advanced cooling technologies that are currently used in USA and EUROPE for the power generation systems as well as aerospace projects.

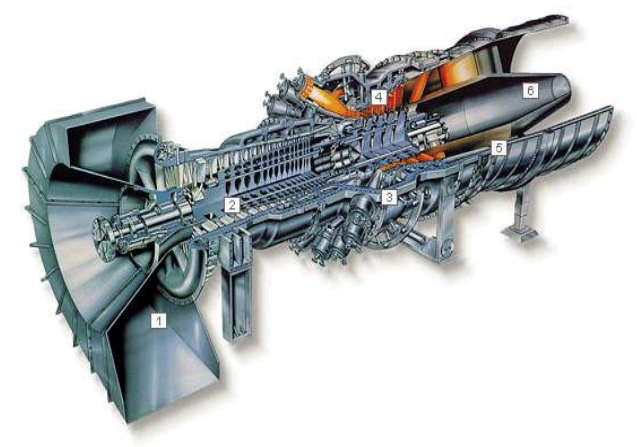

Figure 1: $\quad$ Combustor and high-pressure turbine.

Cooling technology, as applied to gas turbine components is composed of five main elements, (1) internal convective cooling, (2) external surface film cooling, (3) materials selection, (4) thermal-mechanical design, and (5) selection and/or conditioning of the coolant fluid. Cooled turbine components are merely highly specialized and complex heat exchangers that release the cold side fluid in a controlled fashion to maximize work extraction. The enhancement of internal convective flow surfaces for the augmentation of heat transfer was initially 
improved some 25 to 30 years ago through the introduction of rib-rougheners or turbulators, and also pin-banks or pin-fins. Figure 2 shows an example schematic of a blade cooling circuit that utilizes many turbulated passages, a pin bank in the trailing edge, and impingement in the leading edge (coolant is released via film holes, tip holes, and trailing edge). These surface enhancement methods continue to play a large role in today's turbine cooling designs. Film cooling is the practice of bleeding internal cooling flows onto the exterior skin of the components to provide a heat flux reducing cooling layer, as shown by the many holes placed over the airfoil in Figure 2. Film cooling is intimately tied to the internal cooling technique used in that the local internal flow details will influence the flow characteristics of the film jets injected on the surface.

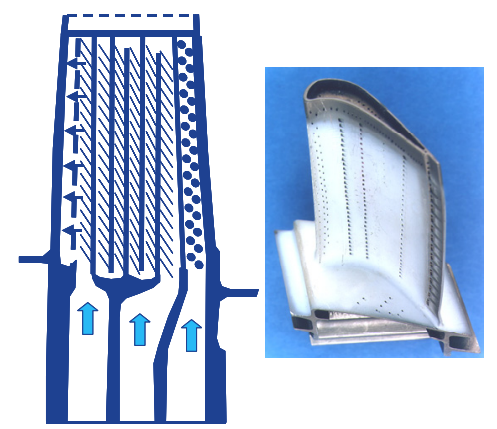

Figure 2: Example cooled turbine blade and cooling circuit.

Several characteristics of gas turbine cooling are worthy of note prior to describing any specific technologies. Almost all highly cooled regions of the high-pressure turbine components involve the use of turbulent convective flows and heat transfer. Very few if any cooling flows within the primary hot section are laminar or transitional. Moreover, the typical range of Reynolds numbers for cooling techniques, using traditional characteristic lengths and velocities, is from 10,000 to 60,000 . This is true of both stationary and rotating components. The enhancement of heat transfer coefficients for turbine cooling makes full use of the turbulent flow nature by seeking to generate mixing mechanisms in the coolant flows that actively exchange cooler fluid for the heated fluid near the walls. These mechanisms include shear layers, boundary layer disruption, and vortex generation. In a marked difference from conventional heat exchangers, most turbine cooling means do not rely on an increase in cooling surface area, since the available surface area to volume ratios are very small. Surface area increases are beneficial, but are not the primary objective of enhancements. The use of various enhancement techniques typically results in at least $50 \%$ and as much as $300 \%$ increase in local heat transfer coefficients over that associated with fully developed turbulent flow in a smooth duct.

Numbers of researchers have been attaching the problem of improving the cooling technologies on these issues. As a demonstration, the author is presenting recent developed computational studies in the blade cooling computations in the following sections. 


\section{Internal cooling}

Three turbulence models, $k-\varepsilon$, Linear $k-\omega$, and RSM were used to predict the streamwise mean velocity $(\mathrm{m} / \mathrm{s})$ distributions. Figure 3 shows streamwise mean velocity contours at Mid-Plane for $\mathrm{Re}=36,000$, where the flow separation occurs at $45^{\circ}$ in the curvature of the bend. All models show some vortex formation at the corners. This is due to the geometry. The flow separation starts at around $45^{\circ}$, the location of the reattachment points change, for RSM this point is around 24 times the curvature diameter. For $k-\varepsilon$, the point is about 14 times the curvature diameter. For $\mathrm{k}-\omega$, this model shows that there are two vortexes; one occurs about 10 times the curvature diameter and second occurs about 20 times the diameter. The secondary flow gradually disappears around 22 times the curvature diameter

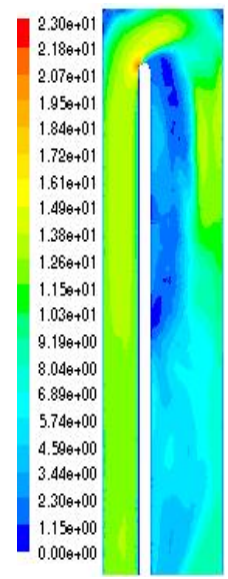

(a)

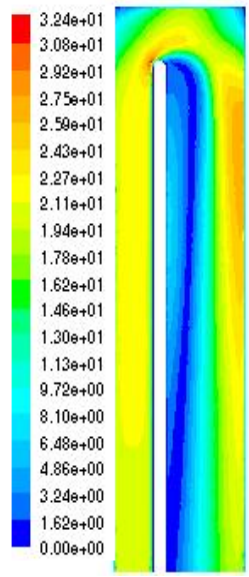

(b)

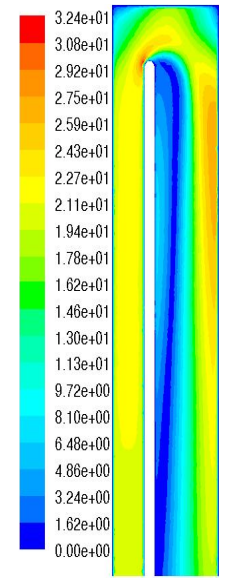

(c)

Figure 3: $\quad$ Mean flow along the mid-plane (m/s) (a) RSM (b) $k-\varepsilon$ and (c) $k-\omega$ at $\mathrm{Re}=36000$.

Depending on the Reynolds number, the flow strength changes causing different separation zones. The flow after the bend is not free to move but is bounded by the outer wall. This causes the fluid to bounce off the wall. This occurs sooner for higher Reynolds number.

All three models predict nearly a similar trend of the Nusselt number, higher in the bend and reducing gradually to downstream. The Nusselt number development in the upstream section is similar and there is an enhancement in Nusselt number within and after the bend, reaching its highest value. This implies that, as is well established in others cases, within the bend, the flow separation, flow impingement and strong cross duct motion, not only cause a reduction in the thickness of the viscous sub-layer, but also reduce its sensitivity to Reynolds numbers. At the downstream section the normalized Nusselt number 
decreases because the secondary flows gradually reduce, also due to increase in cross section, which causes a decrease in the Reynolds number.

The k- $\omega$ model is poor in capturing high turbulence flow as can be observed. Figure 4 shows the mid-plane mean velocity distribution in the vector form, for experimental [1] and the three models at $\mathrm{Re}=36,000$. The agreement between the experiment and the computation is well, in particular quite matches with the computations using RSM. The curvature-induced separation bubble along the inner wall is considerably smaller than the observed by Cheah et al. [3]. Because of the present geometry, the effective cross sectional area reduces over the second half of the bend, imposing an overall acceleration of the flow. However, additional separation bubbles are formed in the corners.

Figure 5 shows the contour normalized Nusselt number for the Experiment [2] and for the three models at $\mathrm{Re}=36,000$. These comparisons are also done in

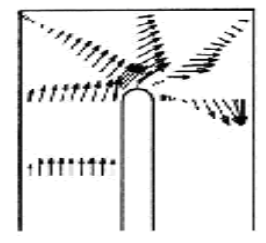

(a)

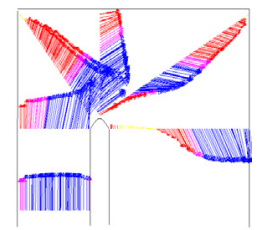

(b)

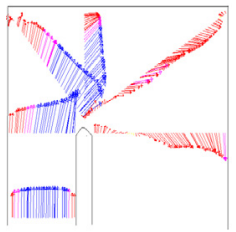

(c)

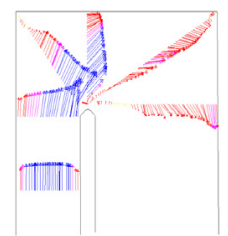

(d)

Figure 4: $\quad$ Mean flow along the mid-plane (m/s) Exp. (b) k- $\varepsilon$ (c) k- $\omega$ (d) RSM at $\mathrm{Re}=36,000$.

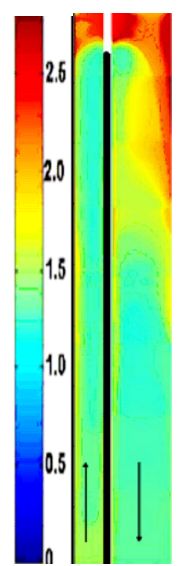

(a)

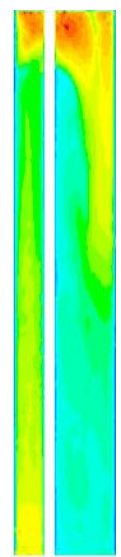

(b)

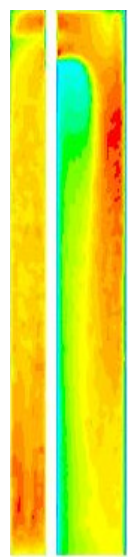

(c)

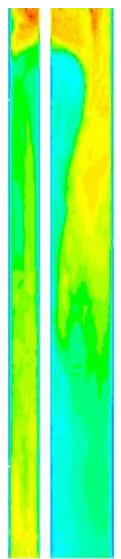

(d)

Figure 5: Nusselt number contour, normalized by the Dittus-Boelter correlations for $\mathrm{Nu}$ (a) Exp (b) $k-\omega$ (c) $k-\varepsilon$ (d) $\mathrm{RSM}$ at $\mathrm{Re}=36,000$. 
Fig. 6, which shows the normalized side averaged Nusselt numbers $\left(\mathrm{Nu}_{\mathrm{avg}} / \mathrm{Nu}_{\mathrm{o}}\right)$ plots at $\mathrm{Re}=36,000$. Here the negative $\mathrm{X} / \mathrm{D}$ refers to upstream and positive refers to downstream. At $\mathrm{X} / \mathrm{D}=0$ the wall is insulated that has high influence on the CFD analysis. This causes deviation observed. As shown in the figures, the agreement is quite good with RSM and also fairly well with $k$ - $\omega$ model. However, the computations with $k$ - $\varepsilon$ model show relatively poor agreement with the experiments.
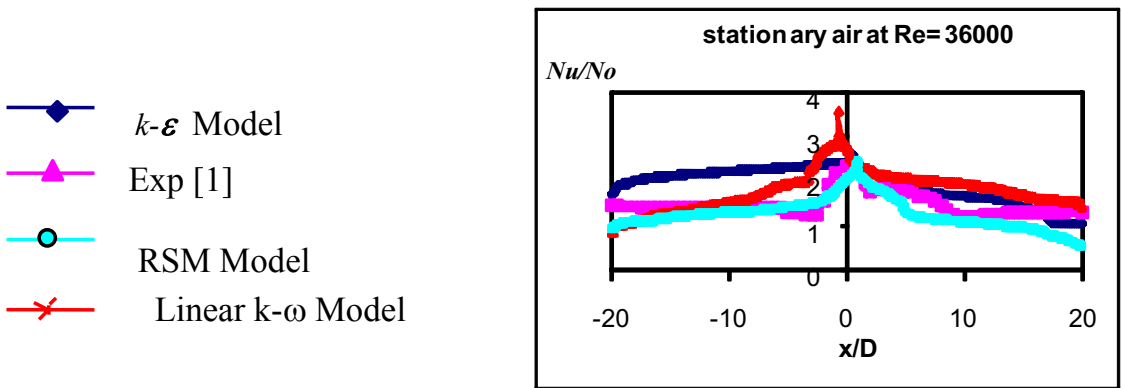

Figure 6: $\quad$ Side-averaged $\mathrm{Nu}$. number for air $(\mathrm{Pr}=0.71)$.

\section{Current trend in cooling technologies}

Figure 7 shows the Nusselt number distribution along the ribbed channels for chevron inline, chevron strips staggered and simple 45 degree angled ribs compared with the smooth channel. As shown in the figure the cases with

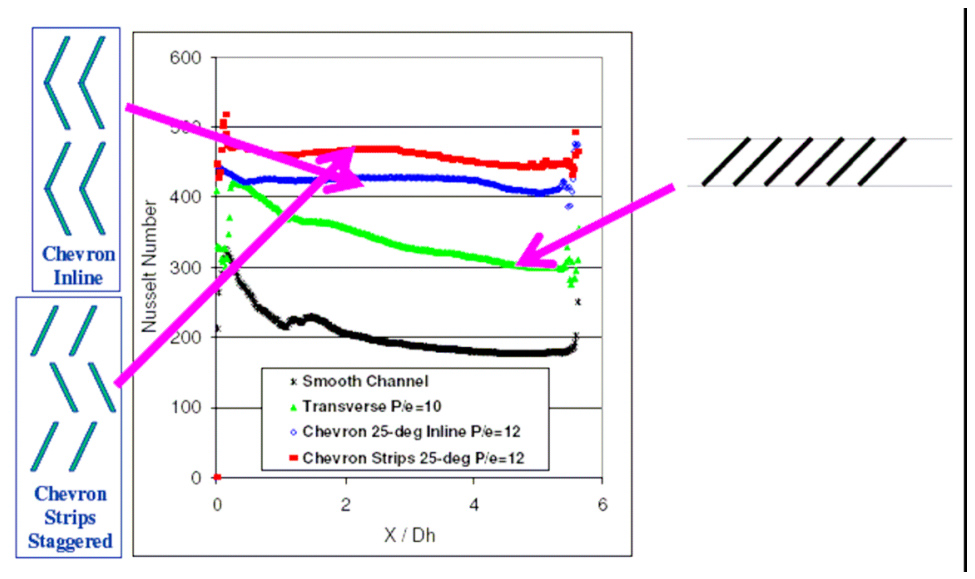

Figure 7: Ribbed channel performance. 
chevron arrangements are in general more effective in keeping the Nusselt number at a higher level throughout the channel.

Full cooling distribution is obtained, and a form of transpiration-like film cooling results from the normal holes in the outer layer. Examples of the overall cooling effectiveness and the film cooling are shown in Fig. 8 by Nakamata et al. [4]. The challenges of this micro cooling include hole plugging, wall strength, film cooling, manufacturing, and cost. No commercial use of these micro cooled solutions has yet appeared.

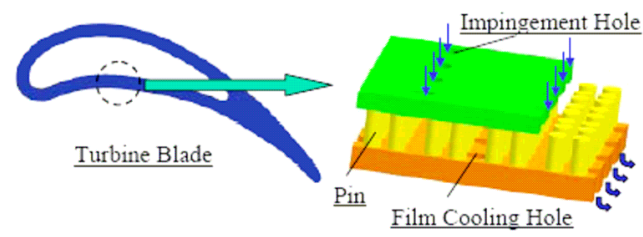

Figure 8: $\quad$ Micro cooling of gas turbine blade [4].

The ultimate goals in gas turbine thermal management include (1) isothermal components to eliminate all internal thermal stresses, (2) hot components to minimize heat loads and inefficient energy transfers in the engine, and (3) minimization of thermal gradients between components and its structures. The advancement of turbine cooling has allowed engine design to exceed normal material temperature limits, but it has shown complexities that have accentuated the heat transfer cooling performances greatly. Cooled component design has consistently trended in the direction of higher heat loads, higher through-wall thermal gradients, and higher in-plane thermal gradients. Gas turbine heat transfer and thermal management technology advancements over the last two decades have primarily come in the more detailed understanding of boundary conditions and the higher sophistication of analysis tools.

Now there are several issues to be considered in further advancing the gas turbine cooling technology. Some technologies such as internal blade cooling and film cooling challenges address active cooling technologies, hot gas flow paths, component design, and systems design. There may not be existing specific solutions, or to prioritize the issues yet as far as the ones to push forward. However, in order to demonstrate some means of comparison, Fig. 9 depicts an approximate ranking of the issues on the basis of technology impact versus technology risk. Such risk-benefit rankings are commonly used to determine a balanced portfolio of funded technology projects within businesses, including research and development. Technology impact includes engine performance metrics and range of product applications, while technology risk refers to the probability of successful development and deployment. The region near the upper-left represents a technology accounting for immediate development; whereas the region near lower-right corner represents the very low benefit-to-risk ratio items that should be avoided. All of these technologies discussed here are seen to cover a broad spectrum of impact and risk, though 


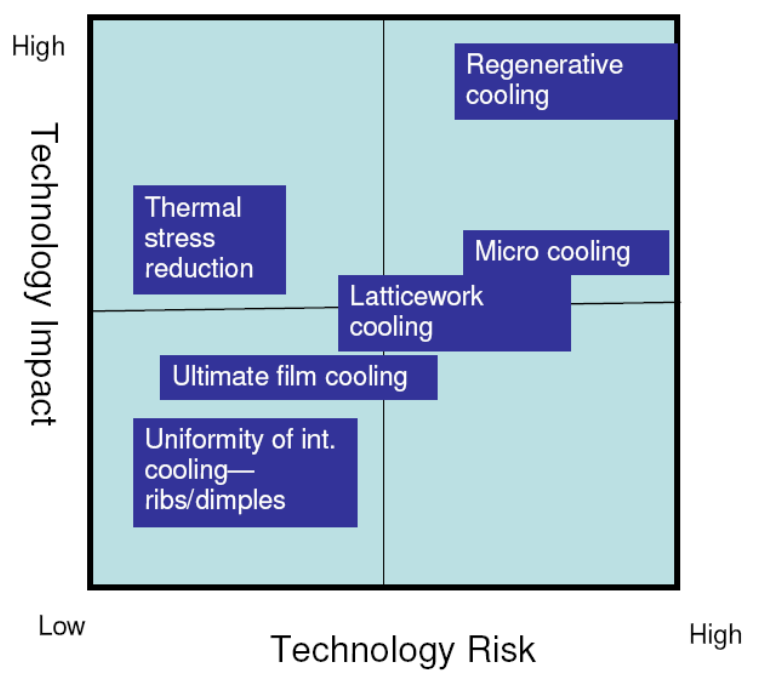

Figure 9: Technology risk vs. technology impact.

individual opinions on rankings may differ. Those items on the left half of the chart mainly represent the issues most strongly being addressed by research today. Those higher risk items on the right side of the chart represent potentially fruitful directions requiring significant innovations and research. Singling out the highest risk-benefit technology identified in Fig. 8, regenerative cooling may cover extensive applications and formats from fairly simple concepts on components to very complex system plant designs. While some versions of regenerative cooling may not entail this high risk, such as those in practice today, this ranking is used to indicate the higher degree of total cycle complexity and risk for most broadly based concepts.

\section{Conclusions}

This summary has presented several of the cutting edges, innovative cooling methods expected to further enhance the aero-thermal-mechanical performance of turbine engines used for power systems. These methods are by no means an exhaustive or comprehensive summary however. Many other variations and combinations of these techniques are anticipated as manufacturing advances become reality. Further improvements and new techniques may become feasible as materials, systems integration, and controls also advance.

Research trends and potential solutions to these thermal issues have been briefly outlined in an attempt to stimulate thought and further ideas. There are no simple solutions for better thermal technologies. It is hoped that this summary will aid in focusing attention in the critical areas of heat transfer and thermal management for gas turbine hot sections required to make significant new gains in overall efficiency, operability, and durability. 


\section{References}

[1] Iacovides, Diamnatis Kounadis and Brian E. Launder "Experimental study of the Thermal Development in a Rotating squared-Ended U-Bend. ASME Turbo Expo 2006: Power Land, Sea and Air. May 2006 GT2006-90846

[2] Iacovides, H., Jackson, D.C., Kelemenis, G., Launder, B.E., Yuan, Y.M., "Experiments on Local Heat Transfer in a Rotating Square-Ended U-Bend," International Journal of Heat and Fluid Flow, Vol. 20, pp. 302-310, 1999.

[3] Cheah S C, Iacovides H, Jackson D C, Ji H and Launder B E., "LDA investigation of the flow development through rotating U-ducts." ASME Journal of Turbomachinery, Vol 118, pp. 590-596, July 1996.

[4] Nakamata, C., Okita, Y., Matsuno, S., Mimura, F., Matsushita, M., Yamana, T., and Yoshida, T., 2005, "Spatial Arrangement Dependence of Cooling Performance of an Integrated Impingement and Pin Fin Cooling Configuration," Paper No. GT2005-68348, IGTI Turbo Expo, Reno-Tahoe, Nevada. 